\title{
Radiation-Induced Glioblastoma Following Radiotherapy for Pituitary Adenomas: Marked Response to Chemotherapy
}

Takashi Kon ${ }^{1,2}$, Manabu Natsumeda ${ }^{1}$, Hitoshi Takahashi ${ }^{3}$, Tomohiko Taki ${ }^{4}$, Yukihiko Fujii $^{1}$, and Ryuya Yamanaka ${ }^{5 *}$

${ }^{1}$ Department of Neurosurgery, Brain Research Institute, Niigata University, Niigata, Japan

${ }^{2}$ Department of Neurosurgery, Kariwa County General Hospital, Niigata, Japan

${ }^{3}$ Department of Pathology, Brain Research Institute, Niigata University, Niigata, Japan

${ }^{4}$ Department of Molecular Diagnostics and Therapeutics, Kyoto Prefectural University of Medicine, Kyoto, Japan

${ }^{5}$ Graduate School for Health Care Science, Kyoto Prefectural University of Medicine, Kyoto, Japan

\begin{abstract}
Radiation-induced glioblastoma is particularly resistant to treatment, and therapeutic options are limited. We report a 48-year-old woman with a radiation-induced glioblastoma who had a complete response to nimustine hydrochloride. Our patient developed a left temporal meninigioma 22 years after and bi-temporal glioblastoma 30 years after a pituitary adenoma was treated with surgery and 50 Gy radiation therapy. She was treated with subtotal resection followed by four cycles of nimustine hydrochloride; a complete response was achieved. She relapsed 16 months after diagnosis of glioblastoma, and underwent further surgery and treatment with temozolomide. She survived for 26 months after the onset of glioblastoma. As the site was within the irradiated area, both meninigioma and glioblastoma were thought to be radiation-induced tumors. If further radiotherapy is not a therapeutic option for glioblastoma, chemotherapy may result in prolonged survival.
\end{abstract}

Keywords: Chemotherapy; Nimustine hydrochloride; Radiationinduced glioblastoma; Single-nucleotide-polymorphism genotyping microarray analysis; Temozolomide

Abbreviations: CNS: Central Nervous System; EGFR: Epidermal Growth Factor Receptor; GBM: Glioblastoma; LOH: Loss of Heterozygosity; MDM2: E3 Ubiquitin Protein Ligase; MGMT: O6methylguanine-DNA methyl-transferase, MTAP: Methylthioadenosine Phosphorylase; PTEN: Phosphatase and Tensin Homolog; PDGFRA: Platelet-Derived Growth Factor Receptor Alpha; SNP: SingleNucleotide-Polymorphism

\section{Introduction}

Radiation-induced central nervous system (CNS) neoplasms are rare, and the cumulative risk of brain tumor after therapeutic cranial irradiation is $0.5-2.7 \%$ at 15 years [1]. Radiation-induced CNS neoplasms are recognized in patients who have had therapeutic radiotherapy to the head or face [2]. Among radiation-induced CNS neoplasms, meningiomas make up about $70 \%$, gliomas about $20 \%$ and sarcomas fewer than 10\% [2-4]. Types of post-radiation gliomas are glioblastoma (GBM) in $75 \%$ and anaplastic astrocytoma in $25 \%$ [1]. Generally, radiation-induced GBM occurs in a younger patient population than would be expected for primary GBM [5]. Radiationinduced GBM is difficult to treat; radiotherapy is not always a therapeutic option because the patient has already been exposed to radiation [6]. We report a patient with a radiation-induced meningioma and GBM who had a sustained remission following chemotherapy alone.

\section{Case Report}

In 1975 a 16-year-old girl was diagnosed with an intra-suprasellar pituitary adenoma. She underwent a biopsy and received conventional radiotherapy with daily fractions of 2 Gy to a total dose of 50 Gy by opposing portal irradiation. She was mentally slow, but functioned and lived independently as an adult. A ventriculoperitoneal shunt had been placed initially and maintained throughout the years. She remained asymptomatic until October 1997 when she developed headache. Cranial magnetic resonance imaging (MRI) revealed a left temporal homogeneously enhancing extra-axial mass. The patient underwent total resection and pathology revealed a fibrous meningioma (Figure 1). She remained asymptomatic until May 2005 when she developed headache. Cranial MRI revealed a bi-temporal heterogeneously enhancing intra-axial mass, with ventricular wall dissemination (Figure $2 \mathrm{~A})$. The patient underwent subtotal resection of the right temporal mass and pathology revealed a GBM (Figure 3A). Post-operatively, the

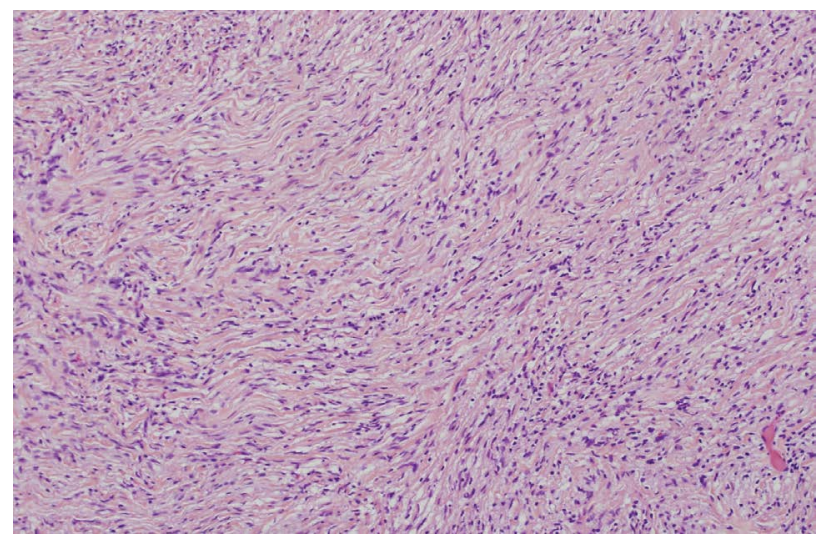

Figure 1: Pathological findings of meningioma. Pathological findings on the tumor removed in 1997. Hematoxylin \& Eosin (H\&E) staining demonstrated fibrous meningioma characterized by parallel fascicles of fibroblastic cells $(\times 100)$.

*Corresponding author: Ryuya Yamanaka, Kyoto Prefectural University of Medicine, Graduate School for Health Care Science, Kyoto 602-8566, Japan, Tel: +81-75-212-5429; Fax: +81-75-212-5423; E-mail: ryaman@cmt.kpu-m.ac.jp

Received July 03, 2013; Accepted July 17, 2013; Published July 25, 2013

Citation: Kon T, Natsumeda M, Takahashi H, Taki T, Fujii Y, et al. (2013) RadiationInduced Glioblastoma Following Radiotherapy for Pituitary Adenomas: Marked Response to Chemotherapy. J Neurol Neurophysiol 4: 155. doi:10.4172/21559562.1000155

Copyright: (c) 2013 Kon T, et al. This is an open-access article distributed under the terms of the Creative Commons Attribution License, which permits unrestricted use, distribution, and reproduction in any medium, provided the original author and source are credited. 
Citation: Kon T, Natsumeda M, Takahashi H, Taki T,Fujii Y, et al. (2013) Radiation-Induced Glioblastoma Following Radiotherapy for Pituitary Adenomas: Marked Response to Chemotherapy. J Neurol Neurophysiol 4: 155. doi:10.4172/2155-9562.1000155

(A)

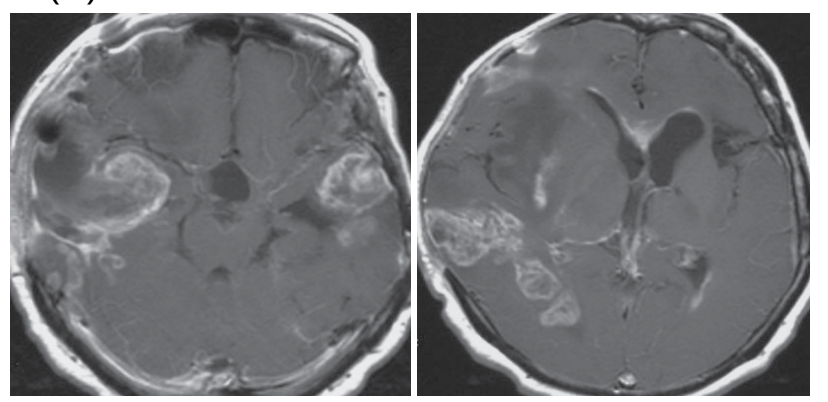

(B)

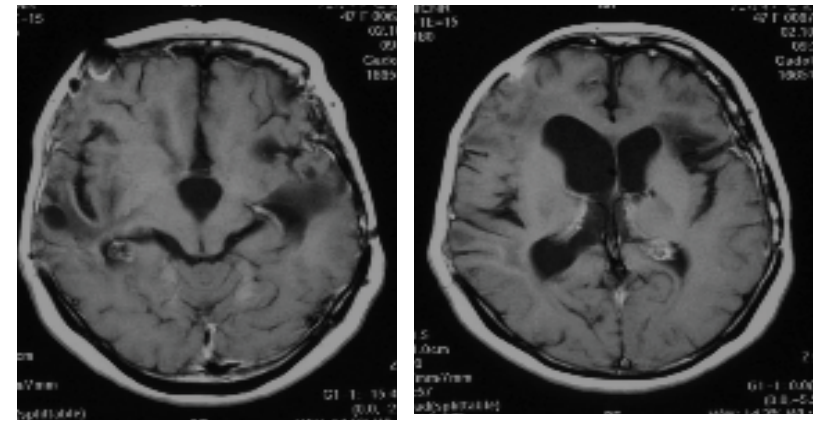

Figure 2: MRI before and after chemotherapy. (A) T1-weighted gadoliniumenhanced magnetic resonance imaging (MRI) from May 2005 demonstrated a well-enhanced mass in bilateral temporal lesion. (B) T1-weighted gadoliniumenhanced MRI from April 2006. No evident tumor after nimustine hydrochloride.

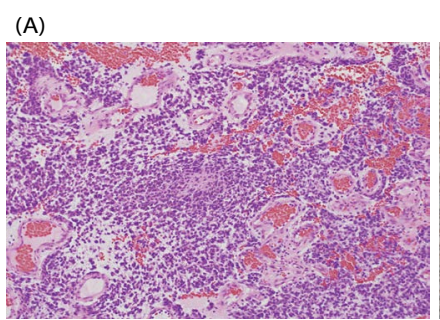

(B)

Figure 3: Pathological findings of glioblastoma. (A) Pathological findings on the tumor removed in 2005. H\&E staining demonstrated necrosis, mitosis, microvasucular proliferation and palisading figures. The histological diagnosis was glioblastoma $(\times 100)$. (B) Immunohistochemical staining of cells for MGMT shows strong immunoreactivity $(\times 100)$.

patient improved and a contrast enhanced MRI of the brain showed bulky residual disease. Further radiation could not be administered safely, and she was started on nimustine hydrochloride (ACNU) (80 $\mathrm{mg} / \mathrm{m}^{2}$ daily every 6-8 weeks). In April 2006, after four cycles, an MRI showed no evidence of disease (Figure 2B), and her Karnofsky Performance Status was 90. Surveillance MRIs showed no disease until September 2006, 16 months from the diagnosis of GBM, when an MRI showed local tumor recurrence; the patient had no symptoms. There were four new nodular areas of enhancement within the surgical cavity of the left parieto-occipital area and linear enhancement of the surgical cavity wall. The patient underwent a repeated craniotomy and pathology again revealed GBM. After surgical removal, she was started on temozolomide ( 5 courses of $80 \mathrm{mg} / \mathrm{m}^{2}$ daily for 21 days every 4 weeks). In July 2007, before the fifth cycle, her chemotherapy was discontinued because of the progressive disease. She died of tumor regrowth in July 2007, 26 months following her first diagnosis of GBM. We analyzed her tumor sample using single-nucleotide-polymorphism genotyping microarray (GeneChip Human Mapping $250 \mathrm{~K}$ Arrays: Affymetrix, Inc., Tokyo, Japan) and O6-methylguanine-DNA methyltransferase (MGMT) immunohistochemistry, according to the method previously described $[7,8]$. On genotyping microarray analysis, loss of heterozygosity (LOH) of 1p, p53, K-ras, p16, methylthioadenosine phosphorylase (MTAP) and amplification of platelet-derived growth factor receptor alpha (PDGFRA), KIT was observed. Amplification and $\mathrm{LOH}$ of epidermal growth factor receptor (EGFR), phosphatase and tensin homolog (PTEN), E3 ubiquitin protein ligase (MDM2) or MGMT were not observed. High MGMT expression was observed on immunohistochemical study (Figure 3B). Informed consent was obtained from the patient in accordance with the guidelines of the Ethical Committee on Human Research, Niigata University Medical School.

\section{Discussion}

The development of brain tumors in previously irradiated sites is a rare complication. Cahan et al. established four criteria to diagnose a radiotherapy-induced brain tumor [9]. These criteria were modified in 1972 by Schrantz [10] as follows: (1) the tumor must appear within the irradiated field; (2) the tumor was not present prior to the radiotherapy; (3) a sufficient latency period must elapse between irradiation and appearance of the tumor; (4) the radiation-induced tumor must be histologically proven and be of a different histological type from the original neoplasm treated by the radiation therapy. In our case, both meningioma and glioblastoma were considered to be radiation induced tumors because both originated within the radiation field. They arose 22 and 30 years after initial irradiation, respectively.

Gliomas are the most frequent glial tumors following sellar region irradiation [11]. Brada et al. described a relative risk of secondary glioma of 7.92 times higher than that of the normal non-irradiated population, with an average latency period to glioma diagnosis of 7 years, in 334 patients with pituitary lesions irradiated to a median dose of $45 \mathrm{~Gy}$ for the sellar region [2]. Tsang et al. estimated the relative risk of glioma occurrence after radiotherapy in patients with pituitary adenomas to be 16 times greater than that of the general population [12]. In cases where the pituitary adenomas are treated by standard fractionated radiation or stereotactic radiosurgery, radiation-induced gliomas should be considered as a possible long-term side effect.

Radiation-induced gliomas are usually high-grade tumors, although low-grade ones $[13,14]$ have been reported. High-grade gliomas are the most common radiation-induced brain tumors observed in children, while benign meningiomas are the most frequently found radiationinduced brain neoplasms in adults [15]. In adults, low-grade gliomas develop after 9.2 years and high-grade gliomas after 12.5 years from the time of irradiation [15]. The relation of the radiation dose to the histological type of the secondary tumor is not proved [15]. In our case, the latency period of 30 years to the occurrence of the radiationinduced glioma is longer than that of reported cases.

Regarding the reports of treatment of radiation-induced glioma, a dramatic response and prolonged survival after treatment with carmustine and temozolomide were reported $[16,17]$. These tumors have a poor prognosis because of their intrinsic resistance to treatment and the difficulty of using aggressive therapies in previously irradiated patients. In our patient, further radiotherapy could not be administered safely. However, the patient had an unexpected complete response to nimustine hydrochloride after subtotal resection. She had an overall survival of 26 months from her diagnosis of GBM. The remarkably durable response to nimustine hydrochloride suggests that vigorous 
Citation: Kon T, Natsumeda M, Takahashi H, Taki T,Fujii Y, et al. (2013) Radiation-Induced Glioblastoma Following Radiotherapy for Pituitary Adenomas: Marked Response to Chemotherapy. J Neurol Neurophysiol 4: 155. doi:10.4172/2155-9562.1000155

chemotherapeutic approaches may yield prolonged disease control in some patients with radiation-induced GBM.

In a patient reported by Gessi et al. the genetic alterations were p53 mutation, LOH of 17 p and 19q, MGMT promoter methylation, and no amplification of EGFR [18]. Nine radiation-induced highgrade gliomas were studied for possible molecular alterations in p53, PTEN, K-ras, EGFR, and p16 by Brat et al [19]. p53 gene mutation is detected in one case, and EGFR amplification and p16/MTAP gene deletion in two cases each [19]. Genetic alterations similar to those described in spontaneous, sporadic primary GBM, except the absence of PTEN mutations, were found in the radiation-induced group. Radiation-induced GBMs have a lower percentage of EGFR and p16 alterations than primary GBM [19]. In our case, although LOH and amplification of EGFR, PTEN was not observed, LOH of 1p, K-ras, p16 and p53 and amplification of PDGFR, KIT were. The relationship of $1 \mathrm{p} \mathrm{LOH}$ and chemosensitivity in oligodendroglial tumors is well known [20]. Although MGMT expression was noted, 1p LOH may account for the marked response to chemotherapy. The molecular alteration of radiation-induced GBM is not well known, owing to the limited number of cases and limited genes analyzed. The marked chemosensitivity should be further investigated for the development of glioma therapy.

\section{Conclusion}

We encountered a patient who presented with glioblastoma 30 years after undergoing partial removal and radiation therapy to treat pituitary adenoma at the identical site in the brain. The remarkably durable response to nimustine hydrochloride was noted. If further radiotherapy is not a therapeutic option for glioblastoma, chemotherapy may be taken into account. The continuing follow-up of pituitary adenoma patients who have undergone radiotherapy will allow early diagnosis of radiation-induced gliomas. At present radiation-induced gliomas must be considered in treatment decision-making in pituitary adenoma patients. Although this clinical course is rare, the possibility of this kind of delayed complication should be considered.

\section{References}

1. Paulino AC, Mai WY, Chintagumpala M, Taher A, Teh BS (2008) Radiationinduced malignant gliomas: is there a role for reirradiation? Int J Radiat Oncol Biol Phys 71:1381-1387.

2. Brada M, Ford D, Ashley S, Bliss JM, Crowley S, et al. (1992) Risk of second brain tumour after conservative surgery and radiotherapy for pituitary adenoma. BMJ 304: 1343-1346.

3. Ron E, Modan B, Boice JD Jr, Alfandary E, Stovall M, et al. (1988) Tumors of the brain and nervous system after radiotherapy in childhood. N Engl J Med 319: 1033-1039.
4. Musa BS, Pople IK, Cummins BH (1995) Intracranial meningiomas following irradiation--a growing problem? Br J Neurosurg 9: 629-637.

5. Simmons NE, Laws ER Jr (1998) Glioma occurrence after sellar irradiation case report and review. Neurosurgery 42: 172-178.

6. Yang SY, Wang KC, Cho BK, Kim YY, Lim SY, et al. (2005) Radiation-induced cerebellar glioblastoma at the site of a treated medulloblastoma: case report. $J$ Neurosurg 102: 417-422.

7. Nakasu S, Fukami T, Baba K, Matsuda M (2004) Immunohistochemical study for O6-methylguanine-DNA methyltransferase in the non-neoplastic and neoplastic components of gliomas. J Neurooncol 70: 333-340.

8. Yamamoto G, Nannya Y, Kato M, Sanada M, Levine RL, et al. (2007) Highly sensitive method for genomewide detection of allelic composition in nonpaired primary tumor specimens by use of affymetrix single-nucleotide-polymorphism genotyping microarrays. Am J Hum Genet 81: 114-126.

9. Cahan WG, Woodard HQ, Higinbotham NL, Stewart FW, Coley EL (1948) Sarcoma arising in irradiated bone; report of 11 cases. Cancer. 1: 3-29.

10. Schrantz JL, Araoz CA (1972) Radiation induced meningeal fibrosarcoma Arch Pathol 93: 26-31.

11. Bonilha L, Borges G, Fernandes YB, Ramina R, Carelli EF, et al. (2000) Pilocytic astrocytoma following radiotherapy for craniopharyngioma: case report. Arq Neuropsiquiatr 58: 731-735.

12. Tsang RW, Laperriere NJ, Simpson WJ, Brierley J, Panzarella T, et al. (1993) Glioma arising after radiation therapy for pituitary adenoma. A report of four patients and estimation of risk. Cancer 72: 2227-2233.

13. Salvati M, Frati A, Russo N, Caroli E, Polli FM, et al. (2003) Radiation-induced gliomas: report of 10 cases and review of the literature. Surg Neurol 60: 60-67.

14. Huang $\mathrm{Cl}$, Chiou WH, Ho DM (1987) Oligodendroglioma occurring after radiation therapy for pituitary adenoma. J Neurol Neurosurg Psychiatry 50 1619-1624.

15. Pettorini BL, Park YS, Caldarelli M, Massimi L, Tamburrini G, et al. (2008) Radiation-induced brain tumours after central nervous system irradiation in childhood: a review. Childs Nerv Syst 24: 793-805.

16. Nicolardi L, DeAngelis LM (2006) Response to chemotherapy of a radiationinduced glioblastoma multiforme. J Neurooncol 78: 55-57.

17. Monje ML, Ramakrishna NR, Young G, Drappatz J, Doherty LM, et al. (2007) Durable response of a radiation-induced, high-grade cerebellar glioma to temozolomide. J Neurooncol 84: 179-183.

18. Gessi M, Maderna E, Guzzetti S, Cefalo G, Massimino M, et al. (2008) Radiation-induced glioblastoma in a medulloblastoma patient: a case report with molecular features. Neuropathology 28: 633-639.

19. Brat DJ, James CD, Jedlicka AE, Connolly DC, Chang E, et al. (1999) Molecula genetic alterations in radiation-induced astrocytomas. Am J Pathol 154: 14311438

20. Thiessen B, Maguire JA, McNeil K, Huntsman D, Martin MA, et al. (2003) Loss of heterozygosity for loci on chromosome arms $1 p$ and $10 q$ in oligodendroglial tumors: relationship to outcome and chemosensitivity. J Neurooncol 64: 271 278 